\title{
Genome-wide analysis of rice cis-natural antisense transcription under cadmium exposure using strand-specific RNA-Seq
}

\author{
Youko Oono $^{1 *+} \mathbb{D}$, Takayuki Yazawa ${ }^{1 \dagger}$, Hiroyuki Kanamori $^{1}$, Harumi Sasaki ${ }^{1}$, Satomi Mori ${ }^{1}$ and Takashi Matsumoto ${ }^{1,2}$
}

\begin{abstract}
Background: The elucidation of novel transcripts and their expression in response to various stress conditions is necessary to understand the transcriptional network of plants as an adaptation to biotic and abiotic stresses. We performed strand-specific RNA-Seq (ssRNA-Seq) on rice exposed to cadmium (Cd) for $24 \mathrm{~h}$ and investigated the expression of cis-natural antisense transcripts (cis-NATs), a class of endogenous coding or non-protein-coding RNAs with sequence complementarity to the opposite strands of RAP transcripts.
\end{abstract}

Results: Many RAP transcripts possessed cis-NATs and these cis-NATs were responsive to some extent. Cis-NATs were upregulated from 26, 266 and 409 RAP gene loci, while 2054, 2501 and 2825 RAP transcripts were upregulated from 38,123 RAP loci under high Cd exposure in roots at 1, 12 and $24 \mathrm{~h}$, respectively. In addition, most of the upregulated cis-NATs showed little upregulation under ABA or cold treatment. A number of cis-NATs were upregulated from less than 35 RAP gene loci in different tissue and time-point combinations under low $\mathrm{Cd}$ exposure, suggesting that cisNATs respond to environmental stress. Furthermore, 409 RAP transcripts with upregulated cis-NATs were classified into three groups based on the expression of the RAP transcripts from the opposite DNA strand, including 138 upregulated, 128 invariable, and 143 downregulated transcripts, although the responses of cis-NATs and RAP transcripts were not always correlated.

Conclusions: We have shown that the cis-NATs identified by ssRNA-Seq analysis are novel genes and that some of them are stress-specific and show different responses depending on the degree of stress and tissue. These results improve our understanding of the complete molecular mechanism of plant adaptation to Cd exposure.

Keywords: Strand-specific RNA-Seq (ssRNA-Seq), cis-natural antisense transcript (cis-NATs), Cadmium (Cd), Abiotic stress, Rice, Transcriptome

\section{Background}

Rice (Oryza sativa L.) is a major staple in many parts of the world. The transcriptomic network of rice under various environmental stress conditions remains to be fully elucidated despite many studies based on RNA sequencing technology, which can accurately quantify gene expression levels over a broad dynamic range and detect transcripts expressed at very low or very high levels, as well as subtle changes [1-3]. This is because of the complexity of the transcriptome including the

\footnotetext{
* Correspondence: yoono@affrc.go.jp

${ }^{\dagger}$ Equal contributors

'Institute of Crop Science, National Agriculture and Food Research

Organization, 2-1-2 Kannondai, Tsukuba, Ibaraki 305-8602, Japan

Full list of author information is available at the end of the article
}

existence of cis-natural antisense transcripts (cis-NATs), a class of endogenous coding or non-protein-coding RNAs with sequence complementarity to the opposite strand of an annotated gene. In addition, many genes have not been functionally characterized, and transcription site boundaries and transcript structures can sometimes change; for example, because of splice isoforms and editing $[4,5]$. The set of transcripts present in a cell, tissue, organ or whole organism also varies at different points in time, and may change depending on the developmental stage or environmental conditions.

We performed a time-course transcriptome analysis of rice (Oryza sativa ssp. japonica cv. Nipponbare) under 140 environmental stress and plant hormone treatment conditions using the RNA-Seq platform to elucidate the 
complete set of transcripts including transcripts showing subtle changes in expression, rare transcripts and variants. The transcripts showing diverse responses under each condition were compiled in the TENOR (Transcriptome ENcyclopedia Of Rice, http://tenor.dna.affrc.go.jp) database [6]. They included a large number of novel transcripts (unannotated transcribed regions with no overlap to any known locus) identified by comparing known transcript structures annotated in the RAP-DB (http://rapdb.dna.affrc.go.jp/) and the MSU Rice Genome Annotation Project database (http://rice.plantbiology.msu.edu/) with Cufflinks-predicted structures. The RNA-Seq technology therefore allows for ultra-deep and highly parallel sequencing of basal transcriptomes under stress conditions and can overcome several limitations of microarray technology.

Recently, strand-specific RNA-Seq (ssRNA-Seq) has become a useful method to define transcriptional orientations and to assess the presence of cis-natural antisense transcripts (cis-NATs). As RNA-Seq analysis does not recognize transcripts transcribed from the opposite DNA strand of the same genomic locus that overlap partly with sense RNA in the same or opposite orientation [7], cis-NATs may have not been identified at all. Large-scale analysis indicates thousands of cis-NATs respond to light in Arabidopsis [8] and heat in Brassica rapa [9], and are expressed in rice under salt, drought and cold stresses, and normal conditions [7]. A few cisNATs have been reported to act as a regulatory class of RNA and can affect transcription or translation by various mechanisms including transcriptional interference. Upregulation of the antisense FLC (FLOWERING LOCUS C, floral repressor gene) transcript may be part of the cold-sensing mechanism, which is correlated with downregulation of the FLC transcript [10]. Downregulation of antisense PHO1;2 (PHOSPHATE1;2) transcript expression resulted in a decrease in the $\mathrm{PHO} 1 ; 2$ protein, while constitutive overexpression of the antisense PHO1;2 transcript led to a strong increase of PHO1;2, even under phosphate-sufficient conditions, suggesting the cis-NAT acts as a translational enhancer for the sense mRNA in rice phosphate homeostasis [11]. However, the existence and responses of cis-NATs as novel transcripts are still not fully understood under various stress conditions.

In the present study, we took advantage of the ssRNASeq method to deeply sequence cDNAs from transcribed RNAs with a clear transcriptional orientation in rice that were associated with the specific stress response to $\mathrm{Cd}$ exposure. To our knowledge, this is the first report of the existence of genes encoding cis-NATs responsive to Cd stress. Only loci overlapping with sense DNA annotated RAP (Rice Annotation Project: http://rapdb.dna.affrc.go.jp/) genes were used to search for cis-NATs.
We focused on cis-NATs from RAP gene loci and excluded the loci of sense-antisense RAP genes, which are transcribed bidirectionally from an overlapping genomic region. Our aim was to explore the existence of cisNATs, which are generally rare, via ssRNA-Seq and to identify novel transcripts expressed specifically under $\mathrm{Cd}$ exposure.

\section{Methods}

\section{Plant culture and treatments}

Rice (Oryza sativa ssp. japonica cv. Nipponbare) seeds were germinated and grown by hydroponic culture in medium containing $1.425 \mathrm{mM} \mathrm{NH} \mathrm{NO}_{3}, 0.323 \mathrm{mM}$ $\mathrm{NaH}_{2} \mathrm{PO}_{4}, \quad 0.513 \mathrm{mM} \quad \mathrm{K}_{2} \mathrm{SO}_{4}, \quad 0.998 \mathrm{mM} \quad \mathrm{CaCl}_{2}$,

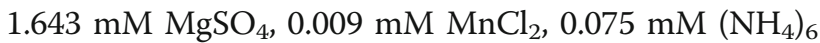
$\mathrm{Mo}_{7} \mathrm{O}_{24}, \quad 0.019 \mathrm{mM} \quad \mathrm{H}_{3} \mathrm{BO}_{3}, \quad 0.155 \mathrm{mM} \quad \mathrm{CuSO}_{4}$, $0.036 \mathrm{mM} \mathrm{FeCl}_{3}, 0.070 \mathrm{mM}$ citric acid, and $0.152 \mathrm{mM}$ $\mathrm{ZnSO}_{4}$ [12]. The seedlings were grown in a growth chamber at $28{ }^{\circ} \mathrm{C}$ under a $16 \mathrm{~h}$ light $/ 8 \mathrm{~h}$ dark cycle with the light period from 6:00 AM to 10:00 PM for 10 days. For $\mathrm{Cd}$ exposure, the seedlings were transferred to a similar culture medium with $0.2,1$ or $50 \mu \mathrm{M} \mathrm{CdSO}$. For $\mathrm{ABA}$ treatment, the seedlings were transferred to culture medium with $100 \mu \mathrm{M} \mathrm{ABA}$. The plants were maintained under each condition for $24 \mathrm{~h}$ at $28{ }^{\circ} \mathrm{C}$ with a $16 \mathrm{~h}$ light $/ 8 \mathrm{~h}$ dark cycle. For cold treatment, the seedlings were transferred to a growth chamber set at $4{ }^{\circ} \mathrm{C}$. Root and shoot samples were collected, frozen in liquid nitrogen, and stored at $-80{ }^{\circ} \mathrm{C}$ until subsequent analyses.

\section{Sequencing and mapping of short reads onto the rice genome}

Total RNA was extracted from all samples using a QIAGEN kit (Invitrogen, Carlsbad, CA, USA) according to the manufacturer's instructions. Strand-specific RNASeq libraries were prepared following the "Directional mRNA-Seq Library Prep Pre-Release" protocol by Illumina. Each sample was sequenced for 75 cycles on an Illumina GAIIx. Low-quality bases $(<\mathrm{Q} 15)$ were trimmed from both the $5^{\prime}$ - and $3^{\prime}$-ends of the reads until a stretch of three or more high-quality $(\geq Q 15)$ nucleotides appeared using an in-house program. Trimming of Illumina adaptor sequences was performed by Cutadapt [13] version 0.9.4. To remove reads from rRNAs, we aligned the reads against rice rRNA gene sequences downloaded from the Rice Annotation Project DataBase (RAP-DB) [14] using Bowtie [15] version 0.12.7, and removed any matching reads. The resulting reads were aligned to the Os-Nipponbare-Reference-The International Rice Genome Sequencing Project-1.0 genome assembly (IRGSP-1.0) (http://rapdb.dna.affrc.go.jp/) using Bowtie, SAMtools version 0.1.18 [16], and TopHat [17] version 1.4.0. The expression level for each transcript was calculated as RPKM (Reads Per Kilobase exon 
Model per Million mapped reads) [18] based on the number of uniquely mapped reads using an in-house program.

\section{Analysis of gene ontology (GO) terms and responsive transcripts under stress treatment}

The GO terms assigned to each transcript were obtained from the RAP-DB for each GO category under the biological process, molecular function, and cellular component domains. Differentially expressed transcripts were detected using a G-test with a FDR (False Discovery Rate) threshold of $0.1 \%$. We focused on genes that had no overlapping genes in the opposite orientation. RPKM values were calculated from at least two biological replicate samples under $\mathrm{Cd}$ exposure with $>0.9$ correlation between them.

\section{Results and discussion}

ssRNA-Seq provides details of the transcriptional structures of cis-NATs transcribed bidirectionally from RAP loci

We generated transcriptome profiles of the early response to $0.2,1$ and $50 \mu \mathrm{M} \mathrm{Cd}$ exposure during plant growth to identify cis-NATs as novel transcripts using ssRNA-Seq, specifically at 1,12 and $24 \mathrm{~h}$. We also generated transcriptome profiles under control conditions at $0,1,12$ and $24 \mathrm{~h}$. In this study, we focused on cis-NATs with sequence complementary to a RAP gene on the opposite DNA strand to identify cis-NATs clearly (Fig. 1a), excluding sense-antisense genes that were transcribed bidirectionally from an overlapping genomic region (Fig. 1b). Because it is hard to distinguish antisense transcripts from sense transcripts transcribed from the same genomic strand in the overlapping genomic region, it can be difficult to accurately quantify the expression level.

\section{Rice genes responsive to $\mathrm{Cd}$ exposure}

$\mathrm{Cd}$ exposure greatly affects rice growth and gene expression even at low concentrations [19]. For each set of conditions, an average of approximately 9.4 million (86.5\%) quality-evaluated reads were mapped to the rice genome sequence and used for further analysis (Additional file 1: Table S1). Analysis of variance (G-test $\{F D R<0.01\})$ on the RPKM-derived read counts was used to dissect the transcriptional responses associated with the time of collection (0, 1, 12 and $24 \mathrm{~h}$ ), hydroponics type (control and $\mathrm{Cd}$ exposure) and tissue (root and shoot). In roots, the number of downregulated RAP transcripts was always greater than that of upregulated

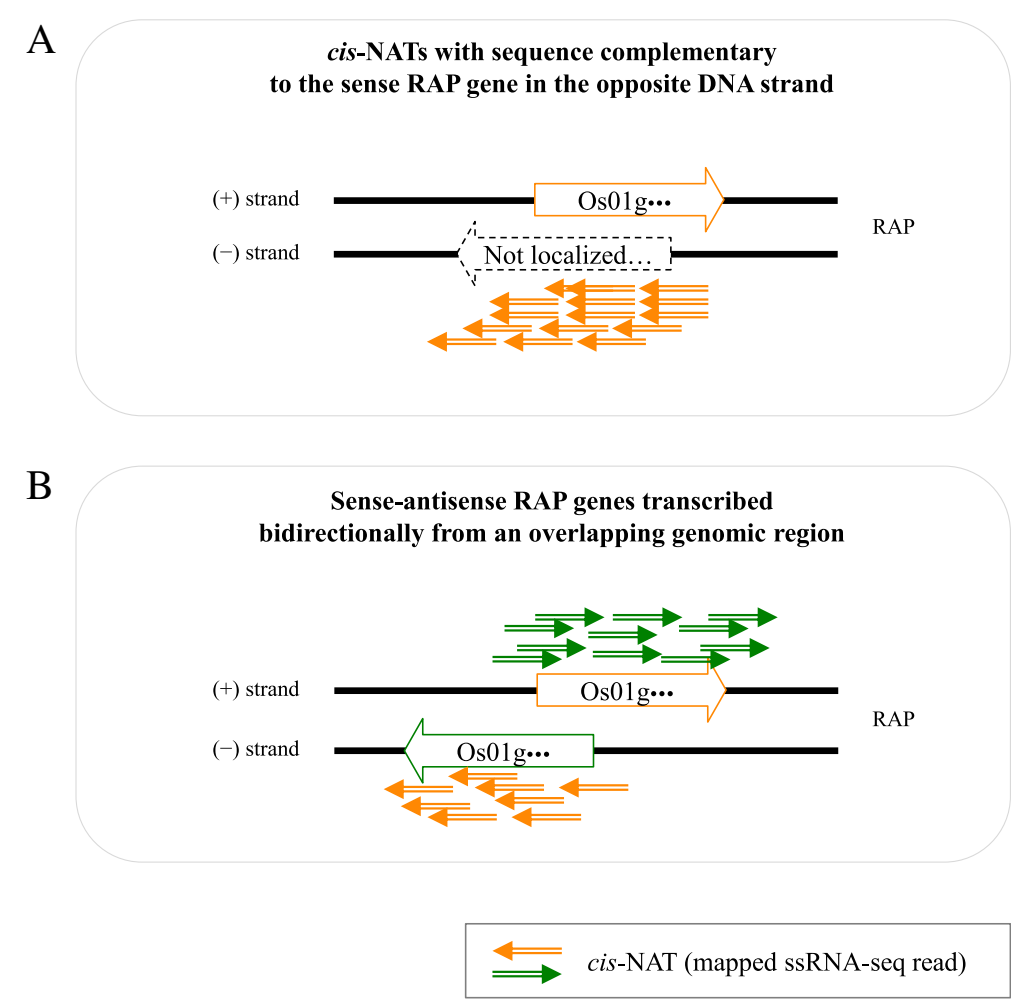

Fig. 1 cis-NATs with sequence complementary to a RAP gene on the opposite DNA strand (a) and sense-antisense RAP genes transcribed bidirectionally from an overlapping genomic region (b). The mapped ssRNA-Seq reads of cis-NATs with sequence complementary to a RAP gene on the opposite DNA strand are indicated by arrows. The origins of reads from overlapping sense-antisense RAP gene loci were difficult to define 
RAP transcripts (Fig. 2), which is the same tendency seen in previous results of RNA-Seq analysis [19]. In shoots, the number of downregulated transcripts gradually increased and reached a maximum under $50 \mu \mathrm{M}$ $\mathrm{Cd}$ at $24 \mathrm{~h}$. The number of responsive transcripts annotated by RAP at $24 \mathrm{~h}$ was $2864,4906,4734$ and 16,567 in roots and 4043, 4155, 3990 and 9315 in shoots, after 0 (control), 0.2, 1 and $50 \mu \mathrm{M} \mathrm{Cd}$ exposure, respectively, compared with non-treatment $(0 \mu \mathrm{M}$ at $0 \mathrm{~h})$ (Fig. 2), suggesting that $50 \mu \mathrm{M} \mathrm{Cd}$ exposure induced responses in more gene responses at $24 \mathrm{~h}$. These data also suggest that the root was more affected by $\mathrm{Cd}$ stress

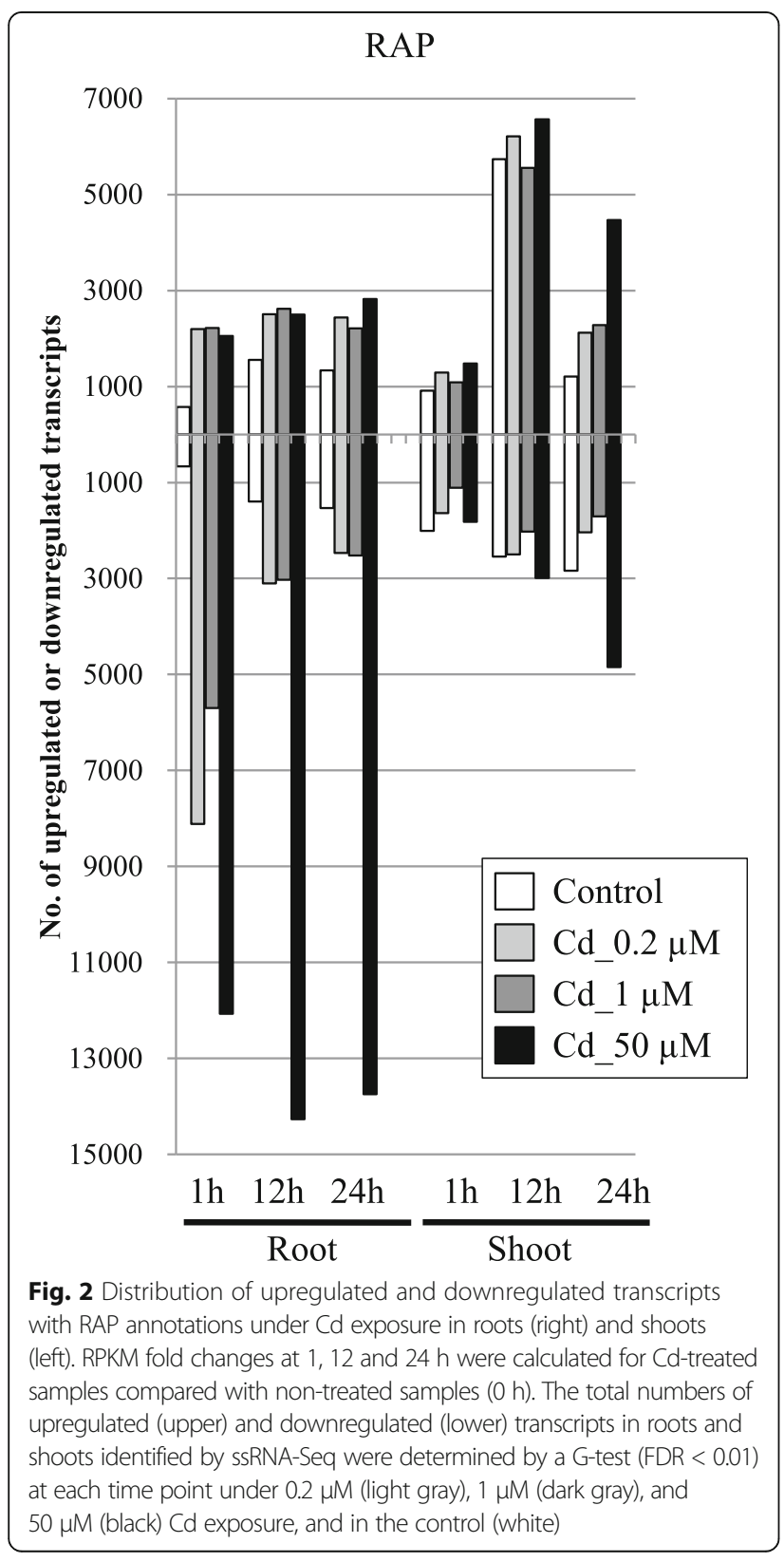

than the shoot at $24 \mathrm{~h}$, which is reasonable because roots are affected by $\mathrm{Cd}$ stress directly. Seventeen metal ion transporters were upregulated $(>5$-fold) under $50 \mu \mathrm{M} \mathrm{Cd}$ at $24 \mathrm{~h}$ (Table 1). The upregulation of seven genes was confirmed under $\mathrm{Cd}$ exposure by qRT-PCR analysis [19], suggesting ssRNA-Seq was successful in identifying Cd-responsive transcripts.

\section{Identification of cis-NATs with sequence complementary to a RAP gene on the opposite DNA strand}

We focused on genomic loci containing a single RAP gene, which included 38,123 RAP gene loci, to define cis-NATs clearly among the 52,640 RAP genes (Fig. 1a). We investigated mapped reads with the opposite orientation in the overlapping genomic region. Among the genomic loci of the 38,123 RAP genes, reads were mapped to 29,323 RAP transcripts (88.0\%) and cis-NATs that were transcribed bidirectionally from 15,588 RAPoverlapping transcripts (40.9\%) in roots, and to 28,626 RAP transcripts (75.1\%) and cis-NATs from 9851 overlapping transcripts $(25.8 \%)$ in shoots at one time point at least under 0 (control), $0.2,1$ and/or $50 \mu \mathrm{M} \mathrm{Cd}$ exposure. This suggested that many single loci with RAP annotations were transcribed bidirectionally from an overlapping genomic region under $\mathrm{Cd}$ exposure, but the cis-NATs themselves were not supported by RAP. Approx. 30\% of all annotated genes were shown to have significant antisense RNA expression in Arabidopsis [20], suggesting that cis-NATs are expressed to some degree in plants and may have diverse transcriptional regulatory mechanisms for carrying out different biological roles from sense transcripts. Huge numbers of unannotated transcripts, which may include cis-NATs previously identified by gene structure predictions [19], are not supported by RAP. Expression of the predicted transcripts from mapped reads was validated by qRTPCR in our previous study [21, 22]. Transcribed extensions and variants from close genes might also have been included. Considering the above, these cis-NATs transcripts are possibly novel transcripts responsive to $\mathrm{Cd}$ exposure in rice, and may include non-protein coding transcripts, novel protein transcripts, transcripts specifically responsive to $\mathrm{Cd}$, or even transcripts that may have lethal functions in E. coli. Analysis of variance (G-test $\{F D R<0.01\}$ ) on the RPKM-derived read counts was used to dissect the responses of cis-NATs based on single RAP gene loci associated with the time of collection $(0,1,12$ and $24 \mathrm{~h}$ ), hydroponics type (control and Cd-treated) and tissue (root and shoot) (Fig. 3). In roots, the number of upregulated cis-NATs gradually increased and reached a maximum under $50 \mu \mathrm{M} \mathrm{Cd}$ at $24 \mathrm{~h}$. This tendency was different from the downregulated transcripts in roots and shoots, and the upregulated transcripts in shoots. The number of upregulated cis- 


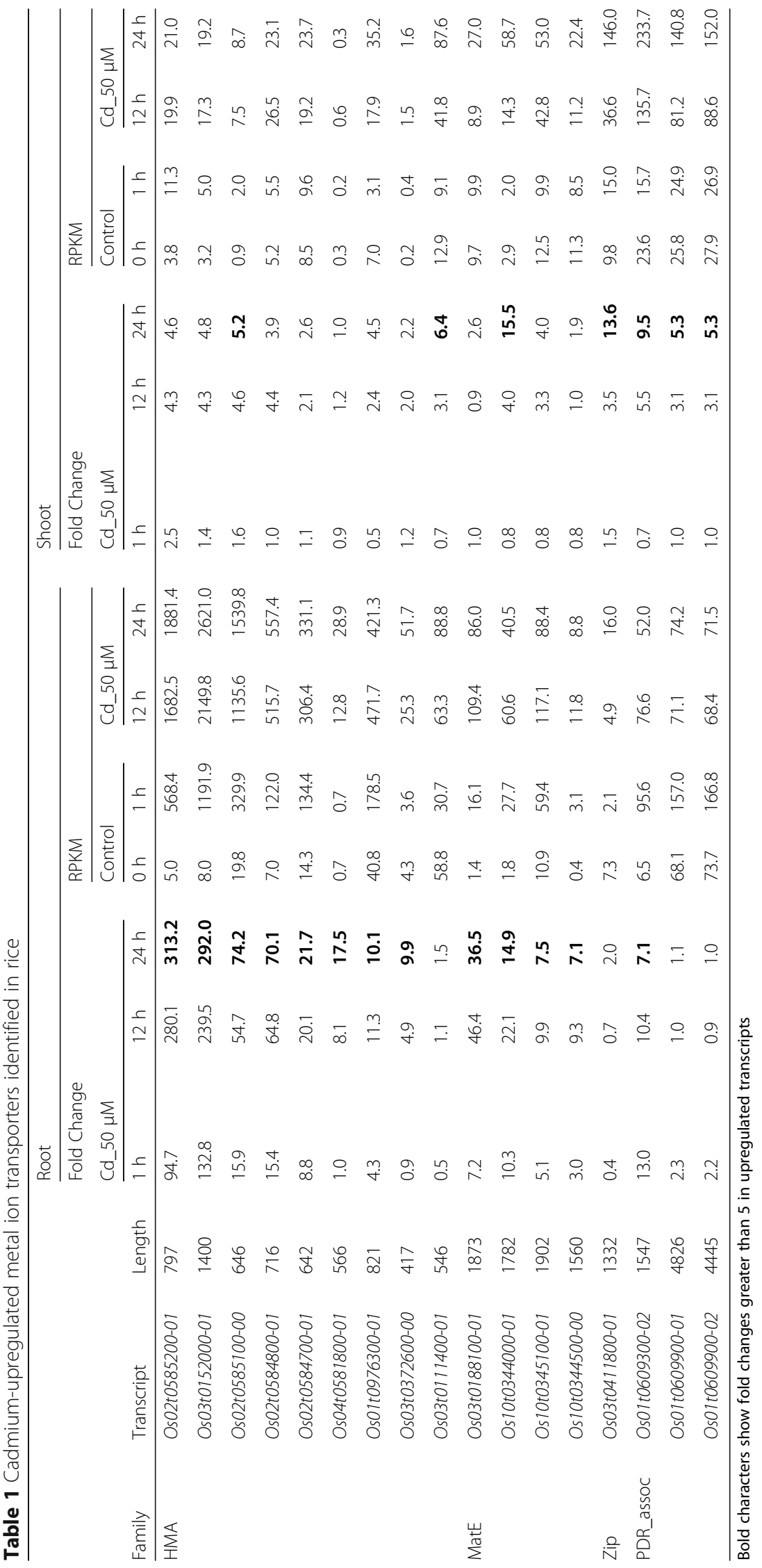




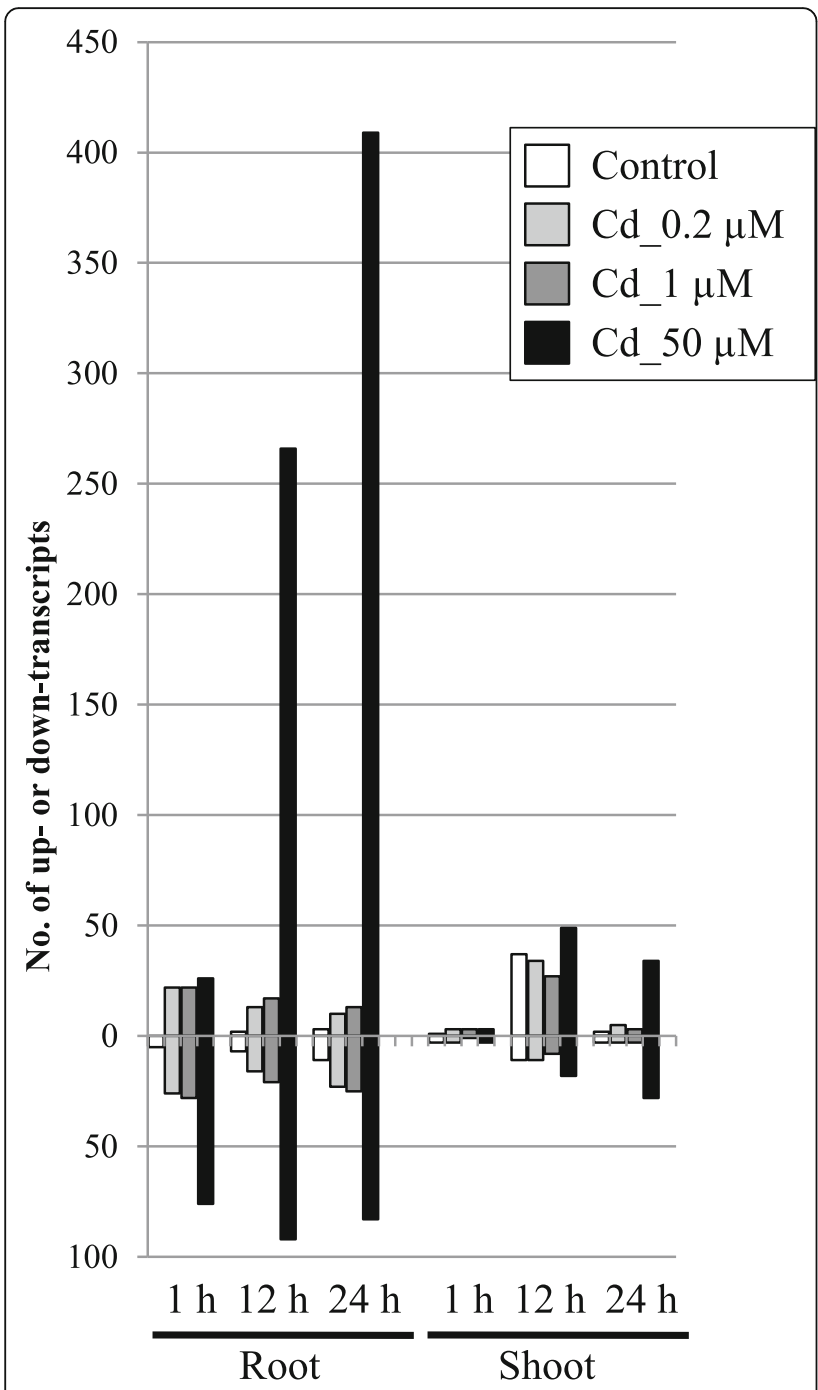

Fig. 3 Distribution of RAP transcripts in roots and shoots with upregulated or downregulated cis-NATs under Cd exposure. RPKM fold changes at 1, 12 and $24 \mathrm{~h}$ were calculated for Cd-treated samples compared with non-treated samples (0 h). The total numbers of upregulated (upper) and downregulated (lower) transcripts in roots and shoots identified by RNA-Seq were determined by a G-test (FDR < 0.01) at each time point under $0.2 \mu \mathrm{M}$ (light gray), $1 \mu \mathrm{M}$ (dark gray), and $50 \mu \mathrm{M}$ (black) Cd exposure, and in the control (white)

NATs originating from single loci annotated by RAP at $24 \mathrm{~h}$ was $14,33,38$ and 409 in roots and 5, 8, 6 and 62 in shoots after $0,0.2,1$ and $50 \mu \mathrm{M} C d$ exposure, respectively, compared with non-treatment $(0 \mu \mathrm{M}$ at $0 \mathrm{~h}$ ) (Fig. 3). Because more cis-NATs responded to the stress at $24 \mathrm{~h}$ as the $\mathrm{Cd}$ concentration increased, the expression of the cis-NATs was affected by the degree of stress. The number of responsive RAP transcripts was much higher than that of cis-NATs (Fig. 2), suggesting that cis-NATs might be rare and respond to specific conditions in rice.
Functional characterization of RAP transcripts with cis-NATs upregulated under $\mathrm{Cd}$ exposure

To determine whether the RAP transcripts with cisNATs had functional tendencies, functional annotations were obtained from the Gene Ontology (GO) database. Functional annotations were assigned to the 409 RAP transcripts with upregulated cis-NATs in roots at $24 \mathrm{~h}$ under $50 \mu \mathrm{M}$ Cd treatment (Fig. 4), because this was the maximum number among the time points (Fig. 3, Table 2 ). The major categories included protein phosphorylation (GO:0006468), regulation of transcription, DNAtemplated (GO:0006355), oxidation-reduction process (GO:0055114) and metabolic process (GO:0008152), a pattern similar to the general stress response. Transcripts for transport (GO:0006810) and transmembrane transport (GO:0055085) were also detected, which clearly validated the RNA-Seq expression profiling data obtained from the rice tissues under $\mathrm{Cd}$ exposure. The upregulation of transport transcripts under $\mathrm{Cd}$ exposure corresponded with the results in Table 1 . The functional categories of the GO classifications were similar between the upregulated RAP transcripts and RAP transcripts with upregulated cis-NATs in roots at 24 h under Cd exposure (Additional file 2: Figure S1). This suggests that the RAP transcripts with upregulated cis-NATs might not have any specific functional tendency compared with the functions of the upregulated RAP transcripts under Cd exposure.

\section{Characterization of the expression of RAP transcripts with cis-NATs under Cd stress}

The functions of RAP transcripts with upregulated cisNATs did not show any specific tendency compared with RAP transcripts. Thus, we investigated the regulation pattern based on the responses of the 409 RAP transcripts with upregulated cis-NATs in roots at $24 \mathrm{~h}$ under $50 \mu \mathrm{M}$ Cd exposure. The transcripts were classified into three sub-groups based on their expression under Cd exposure: 138 RAP transcripts with upregulated cis-NATs were upregulated (ex. Os05t0194500), 128 RAP transcripts with upregulated cis-NATs showed no change (ex. Os04t0407500) and 143 RAP transcripts with upregulated cis-NATs were downregulated (ex. Os01t0949800) (Fig. 5, Additional file 1: Table S2). The upregulation of Os05t0194500 (ONAC085) means that few reads in the sense direction (light blue arrows) were mapped to the Os05g0194500 locus before treatment $(0 \mathrm{~h})$ but the number of reads increased drastically under $\mathrm{Cd}$ exposure. While few reads in the antisense direction (light red arrows) were mapped to the Os05g0194500 locus (AS_Os05t0194500-0 as antisense transcripts with sequence complementarity to Os05t0194500-01 on the opposite DNA strand) at $0 \mathrm{~h}$, they also increased at $24 \mathrm{~h}$ under $\mathrm{Cd}$ exposure (Fig. 5a). This group included many 


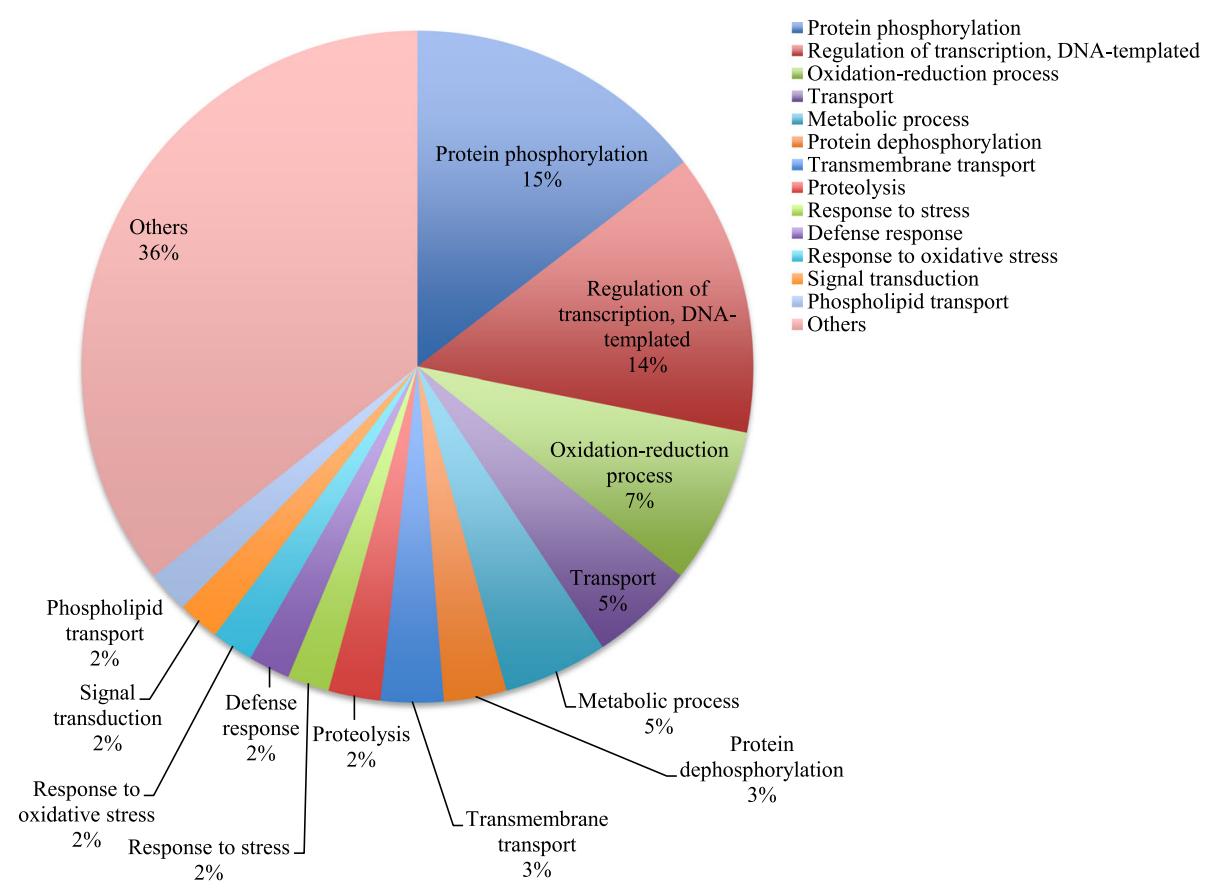

Fig. 4 Distribution of Gene Ontology (GO) biological process categories for RAP transcripts with cis-NATs upregulated under Cd exposure. The percentages of transcripts upregulated in roots after $24 \mathrm{~h}$ of $50 \mu \mathrm{M} C \mathrm{C}$ exposure in different $\mathrm{GO}$ categories are summarized

genes encoding regulatory proteins such as Os01g0749300 (HSFA4A) that confer Cd tolerance [23], and they may function in regulating downstream genes under Cd exposure. Several RAP genes with upregulated cis-NATs encoding functional proteins such as transporters and protective macromolecules (enzymes, protein complexes and membranes) were also included in the group. For example, the gene encoding metal ion transporter (Os04t0533900) may function in efflux pumping of $\mathrm{Cd}$ at the plasma membrane and uptake of $\mathrm{Cd}$ from the soil through the root. The gene encoding sugar transporter (Os03t0218400), a homolog of Arabidopsis STP13 (At5g26340) [24], may function in transporting sugars through plasma membranes to help adjust carbon and nitrogen metabolism during plant growth and development. The gene encoding lipase (Os12t0554500) may function to change the lipid composition of membranes or function to repair stressinduced damage to membranes by regulating permeability to toxic ions and the fluidity of the membrane $[25,26]$. The upregulation of their cis-NATs may contribute to enhancing the expression of the opposite RAP transcripts. The invariability of Os04t0407500 (unknown protein) means that a few reads in the sense direction (light red arrows) were mapped to the Os04g0407500 locus both at $0 \mathrm{~h}$ and under $\mathrm{Cd}$ exposure. While few reads in the antisense direction (light blue arrow) were mapped to the Os04g0407500 locus (AS_Os04t0407500 as antisense transcripts with sequence complementary to
Os04t0407500 on the opposite DNA strand) at $0 \mathrm{~h}$, they drastically increased at $24 \mathrm{~h}$ under $\mathrm{Cd}$ exposure (Fig. 5b). The downregulation of Os01t0949800 (Glutathione S-transferase GST 28: GSTU36) means that many reads in the sense direction (light red arrows) were mapped to the Os01g0949800 locus before treatment $(0 \mathrm{~h})$ but the reads decreased drastically under $\mathrm{Cd}$ exposure. While few reads with antisense direction (light red arrows) were mapped to the Os01g0949800 locus (AS_Os01t0949800 as antisense transcripts with sequence complementary to Os01t0949800 on the opposite DNA strand) at $0 \mathrm{~h}$, they increased at $24 \mathrm{~h}$ under $\mathrm{Cd}$ exposure (Fig. 5c). The GST enzyme catalyzes the conjugation of glutathione to a range of electrophilic substrates for detoxification and protection of the cell [27]. The downregulation of GSTU36 in roots under Cd exposure was consistent with our previous report [19], suggesting it probably functions during normal growth to maintain homeostasis in rice. Functional genes related to plant growth including plant hormone metabolism include genes such as IAA11 (Os05t0559400), which functions in lateral root initiation [28], and ARF23 (Os11t0523800), which functions in auxin-mediated cell growth by promoting RMD (rice actin-binding protein) expression [29], were also included in this group. The transcriptional genes in this category were negatively correlated with cis-NAT accumulation. Cisantisense overlapping pairs have the potential to generate 


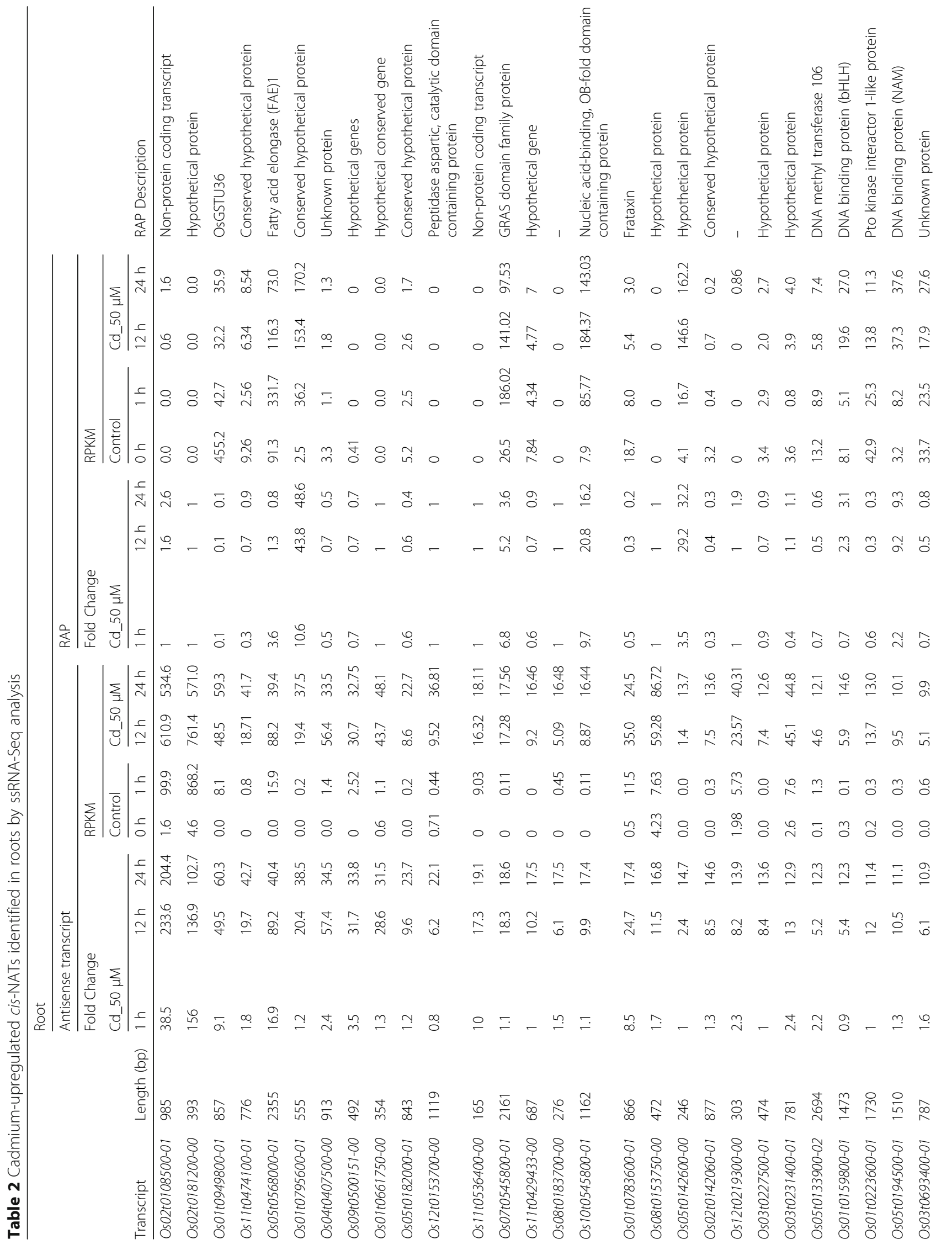




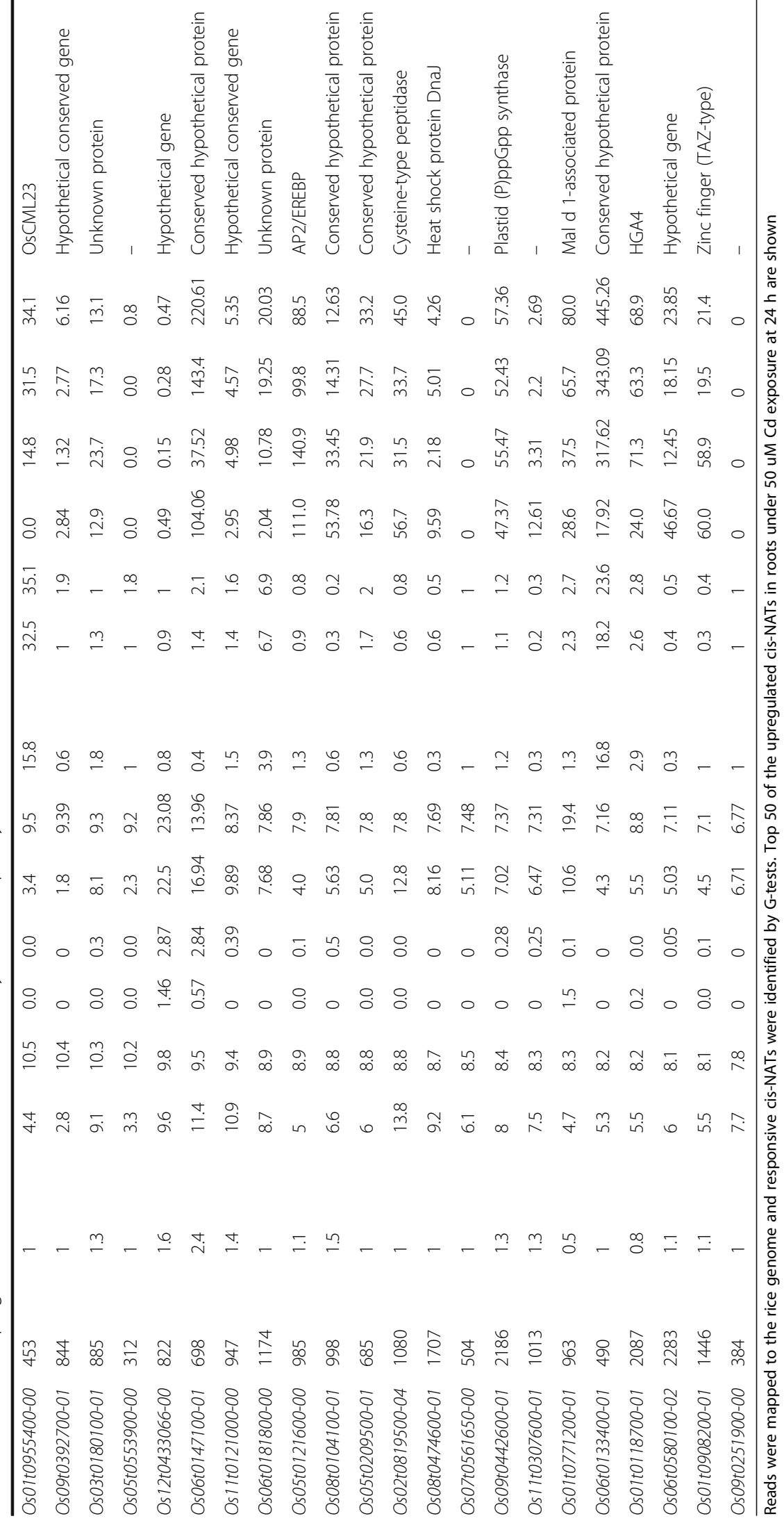




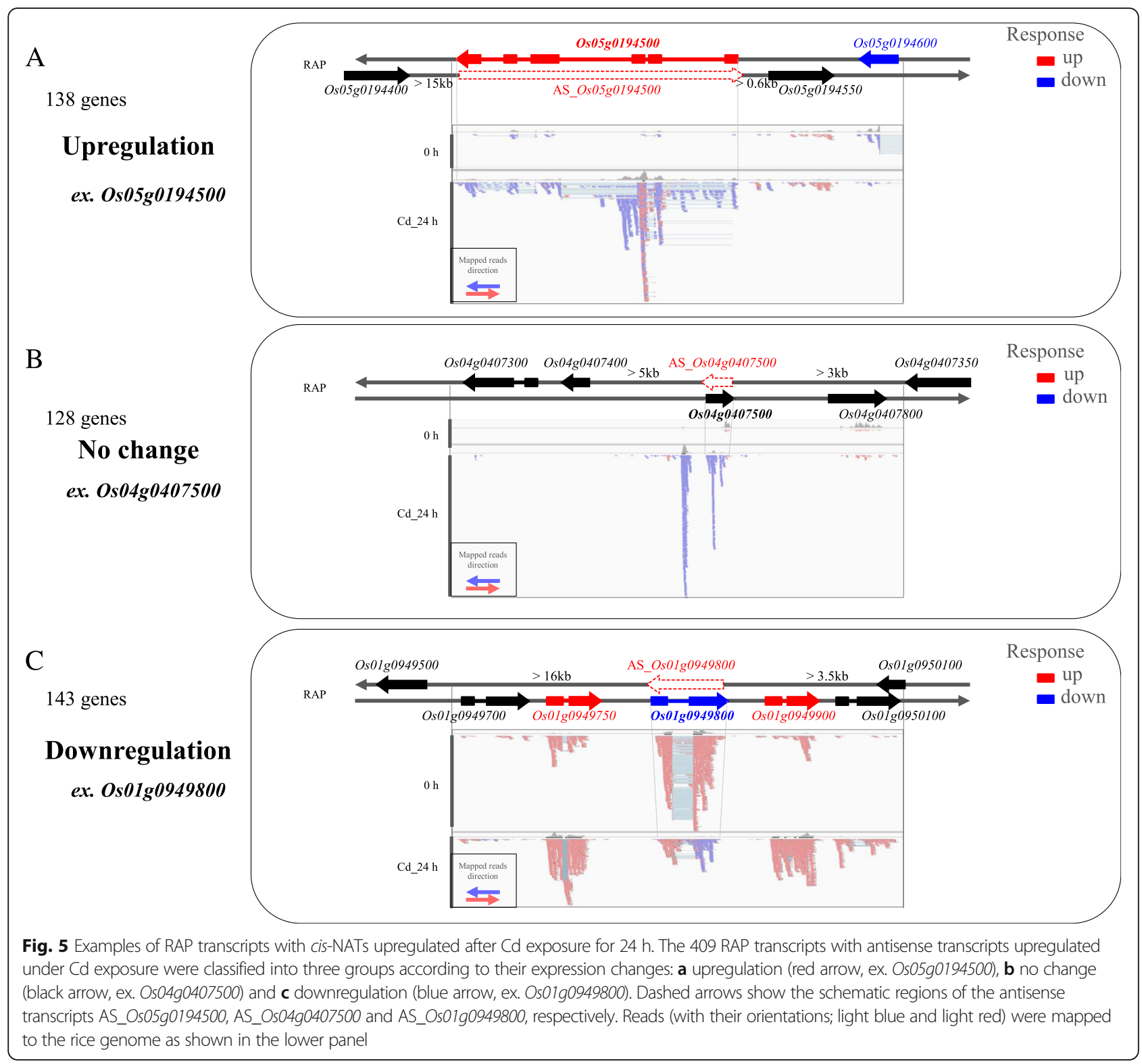

nat-small RNAs, which originate from double-stranded RNA molecules [30], whose action might be contributing to the correlation with quick transcriptional changes, especially in response to stress.

The loci of the cis-NATs with sequence complementary to RAP genes on the opposite DNA strand were more than $3 \mathrm{~kb}$ away from the adjacent RAP genes (Fig. 5). Although the expression of cis-NATs complementary to RAP genes on the opposite DNA strand changed under $\mathrm{Cd}$ exposure, it is difficult to define the exact lengths of the cis-NATs and their loci because they may include splicing isoforms and variants of adjacent genes. Our data clearly indicated that the 409 RAP transcripts with upregulated cis-NATs in roots after $24 \mathrm{~h}$ of $50 \mu \mathrm{M} \mathrm{Cd}$ exposure could be roughly divided into three sub-groups, suggesting that the expression of each RAP transcript might not always be correlated with the upregulated cis-NAT. ssRNA-Seq analysis revealed novel transcripts that were responsive to some degree (ex. $\approx$ 1\%: 409 RAP transcripts with upregulated cis-NATs/ 38,123 expressed RAP genes in roots at $24 \mathrm{~h}$ under $50 \mu \mathrm{M} \mathrm{Cd}$ exposure), suggesting that this method can contribute to gene annotation and improve our understanding of the transcriptome network in rice.

\section{Identification of stress-specific cis-NATs under cold and ABA treatments}

Generally, many genes show specific expression under abiotic stresses, so the responsive cis-NATs were investigated under cold stress and ABA treatment, and in a control 
after $24 \mathrm{~h}$. The cis-NATs showed greater up- or downregulation in roots compared with shoots in all conditions including the control (Fig. 6a). Compared with nontreatment, the cis-NATs were more responsive under stress conditions than in the control after $24 \mathrm{~h}$. In particular, cold-stress conditions had the biggest effect on the cisNAT expression profile, with the most upregulated transcripts in roots and shoots among the conditions (Fig. 6a). Interestingly, the number of RAP transcripts upregulated under cold stress was lower than that under $\mathrm{Cd}$ and $\mathrm{ABA}$, suggesting that the expression tendencies of RAP transcripts and cis-NATs were diverse with regard to stresses (Fig. 6a, Additional file 2: Figure S2A). The number of up- or downregulated RAP transcripts in shoots was greater than that in roots in some conditions (Additional file 2: Figure S2A). Venn diagram analysis of the upregulated cis-NATs among stress conditions showed that most were expressed specifically in one condition in both roots and shoots (Fig. 6b). Additionally, 7.8\% (32/409) of the upregulated cis-NATs under $\mathrm{Cd}$ in roots and $2.9 \%(10 / 34)$ of those in shoots were commonly upregulated by cold and/or ABA. Only one cis-NAT was upregulated under all stress conditions in both roots and shoots (Fig. 6b). Additionally, 54.7\% $(1546 / 2825)$ of the upregulated RAP transcripts under $\mathrm{Cd}$ in roots and $64.7 \%(2892 / 4471)$ of those in shoots were commonly upregulated by cold and/or ABA (Additional file 2: Figure S2B). Meanwhile, 53.0\% (44/83) of the downregulated cis-NATs under $\mathrm{Cd}$ in roots and $57.1 \%(16 / 28)$ of those in shoots, and $68.5 \%(9418 / 13,742)$ of the downregulated RAP transcripts under $\mathrm{Cd}$ in roots and $89.3 \%$ $(4326 / 4844)$ of those in shoots were commonly downregulated by cold and/or ABA (Additional file 2: Figure S3A, B). This indicated that, like the RAP transcripts, the cis-NATs identified by ssRNA-Seq analysis had stress-specific responses.

\section{Conclusion}

Our data encompass the expression of cis-NATs with sequence complementarity to RAP genes on the opposite DNA strand. The cis-NAT loci were defined by their transcriptional structures as revealed by ssRNA-Seq, but
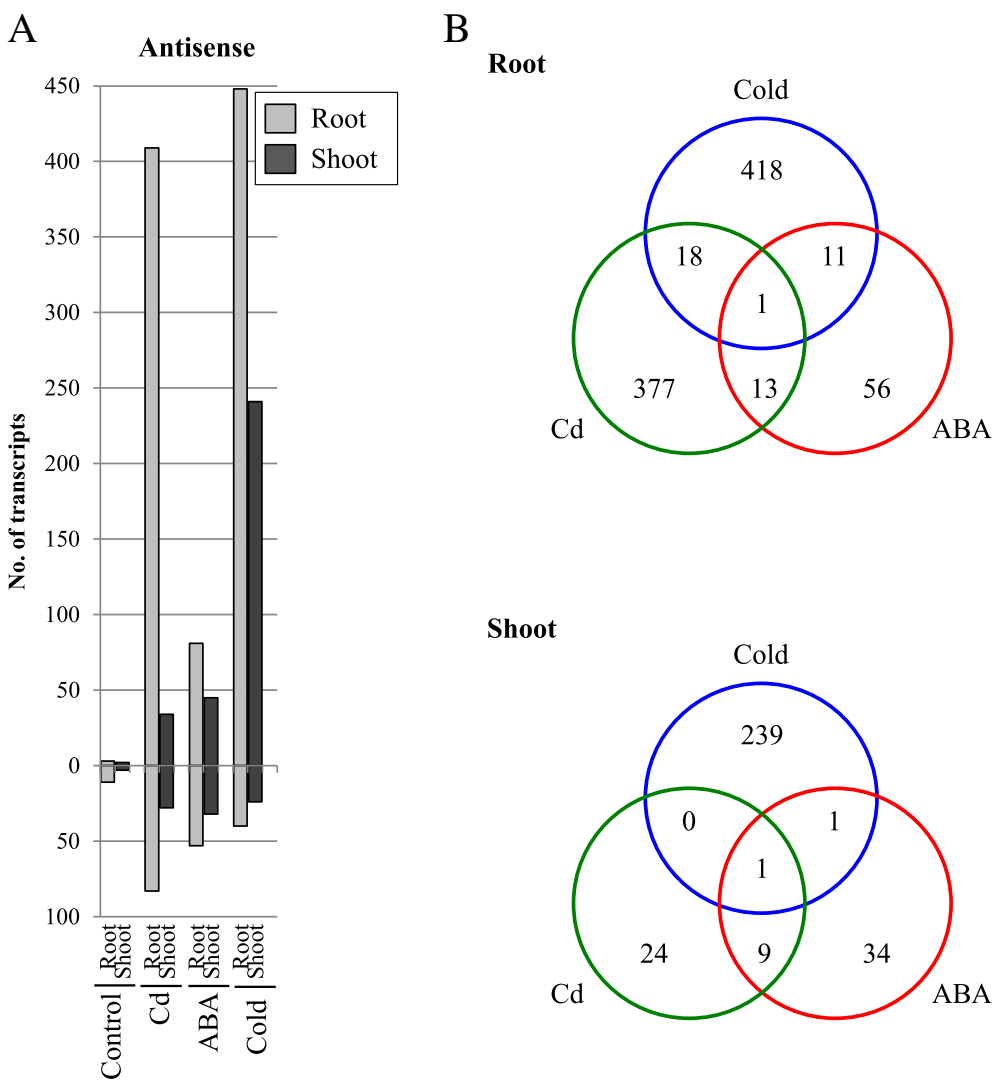

Fig. 6 Distribution of upregulated and downregulated RAP transcripts with corresponding upregulated or downregulated cis-NATs under Cd, ABA, cold, and control treatments for $24 \mathrm{~h}$. a RPKM fold changes at $24 \mathrm{~h}$ were calculated for treated samples compared with non-treated samples $(0 \mathrm{~h})$ in roots (light gray) and shoots (gray). The total numbers of upregulated (upper) and downregulated (lower) transcripts in roots and shoots identified by ssRNA-Seq were determined by a G-test (FDR < 0.01). b The Venn diagram shows the number of RAP transcripts with antisense transcripts upregulated under $\mathrm{Cd}$ exposure (green), ABA treatment (red), and cold treatment (blue) 
were not supported by RAP. Many RAP transcripts possessed cis-NATs, which can be classified as novel transcripts in rice. This study also revealed cis-NATs that were responsive to $\mathrm{Cd}$ exposure to some extent and some that were stress-specific. The numbers of responsive cis-NATs and RAP transcripts on the opposite strand both changed depending on the combination of tissue, $\mathrm{Cd}$ concentration and time point. Future analysis of the exact loci of the transcribed cis-NATs and the timing of their expression will provide useful knowledge for understanding the transcriptome network in rice under $\mathrm{Cd}$ exposure.

\section{Additional files}

Additional file 1: Table S1. Mapping of RNA-Seq reads obtained from root and shoot samples to the reference IRGSP-1.0 genome sequence. Table S2. Classification of 409 RAP transcripts with upregulated cis-NATs into three groups based on the expression of transcribed RAP transcripts from the opposite DNA strand. (XLS $367 \mathrm{~kb}$ )

Additional file 2: Figure S1. Distribution of Gene Ontology (GO) biological process categories for RAP transcripts upregulated under $\mathrm{Cd}$ exposure. The percentages of upregulated transcripts in roots after $24 \mathrm{~h}$ of $50 \mu \mathrm{M} C d$ exposure in different $\mathrm{GO}$ categories are summarized. Figure S2. Distribution of upregulated RAP transcripts. (A) The numbers of upregulated and downregulated RAP transcripts under $\mathrm{Cd}, \mathrm{ABA}$ and cold treatments, and in the control after $24 \mathrm{~h}$ in roots (light gray) and shoots (dark gray). RPKM fold changes at $24 \mathrm{~h}$ were calculated for treated samples compared with non-treated samples $(0 \mathrm{~h})$. The total numbers of upregulated (upper) and downregulated (lower) transcripts in roots and shoots identified by ssRNA-Seq were determined by a G-test (FDR $<0.01$ ). (B) Venn diagram showing the RAP transcripts upregulated under $\mathrm{Cd}$ exposure (green), ABA treatment (red), and cold treatment (blue). Figure S3. Venn diagram analysis of downregulated RAP transcripts. The numbers of RAP transcripts with cis-NATs (A) and RAP transcripts (B) downregulated under $\mathrm{Cd}$ exposure (green), ABA treatment (red), and cold treatment (blue) are shown. (PPTX $57 \mathrm{~kb})$

\section{Abbreviations}

Cd: Cadmium; cis-NAT: cis-natural antisense transcript; FLC: FLOWERING LOCUS C, floral repressor gene; GO: Gene ontology; IRGSP-1.0: The International Rice Genome Sequencing Project-1.0; PHO1;2: PHOSPHATE1;2); RAP: Rice annotation project; RPKM: Reads Per Kilobase exon Model per Million mapped reads; ssRNA-Seq: Strand-specific RNA-Seq

\section{Acknowledgements}

We thank J. Ogata, F. Aota, K. Ohtsu and K. Yamada for technical assistance. We also thank B. Antonio (NARO International Relations Office) for valuable comments and suggestions.

\section{Funding}

This work was supported a grant from the Ministry of Agriculture, Forestry and Fisheries of Japan (Genomics for Agricultural Innovation, RTR-0001). The funding bodies had no role in the design of the study, collection, analysis and interpretation of data, and in writing the manuscript.

\section{Availability of data and materials}

The resulting mRNA-Seq data are deposited in the DDBJ Sequence Read Archive (Accession No. DRA005733).

\section{Authors' contributions}

YO and TM conceived the study and designed the experiments. TM participated in sampling. HK, HS and SM performed the experiments. YO, TY and HK analyzed the data and contributed the analysis tools. YO wrote the manuscript. TY and TM provided valuable insights in the discussion and revision of the manuscript. All authors read and approved the final manuscript.

\section{Ethics approval and consent to participate}

The rice material (Accession number: 229,579) used in this study was obtained from the Genetic Resources Center, NARO (http://www.naro.affrc.go.jp/english/ ngrc/index.html), and is publicly available for non-commercial purposes.

\section{Consent for publication}

Not applicable.

Competing interests

The authors declare that they have no competing interests.

\section{Publisher's Note}

Springer Nature remains neutral with regard to jurisdictional claims in published maps and institutional affiliations.

\section{Author details}

${ }^{1}$ Institute of Crop Science, National Agriculture and Food Research Organization, 2-1-2 Kannondai, Tsukuba, Ibaraki 305-8602, Japan. ${ }^{2}$ Present address: Laboratory of Plant Molecular Breeding, Department of Bioscience, Tokyo University of Agriculture, 1-1-1 Sakuragaoka, Setagaya-ku, Tokyo 156-8602, Japan.

Received: 28 April 2017 Accepted: 1 September 2017

Published online: 06 October 2017

References

1. Li H, Durbin R. Fast and accurate short read alignment with burrowswheeler transform. Bioinformatics. 2009;25(14):1754-60.

2. He GM, Zhu XP, Elling AA, Chen LB, Wang XF, Guo L, Liang MZ, He H, Zhang HY, Chen FF, et al. Global epigenetic and transcriptional trends among two rice subspecies and their reciprocal hybrids. Plant Cell. 2010; 22(1):17-33

3. Lu TT, Lu GJ, Fan DL, Zhu CR, Li W, Zhao QA, Feng Q, Zhao Y, Guo YL, Li WJ, et al. Function annotation of the rice transcriptome at single-nucleotide resolution by RNA-seq. Genome Res. 2010;20(9):1238-49.

4. Hassan MA, Saeij JP. Incorporating alternative splicing and mRNA editing into the genetic analysis of complex traits. BioEssays. 2014;36(11):1032-40.

5. Wang Z, Gerstein M, Snyder M. RNA-Seq: a revolutionary tool for transcriptomics. Nat Rev Genet. 2009;10(1):57-63.

6. Kawahara Y, Oono Y, Wakimoto H, Ogata J, Kanamori H, Sasaki H, Mori S, Matsumoto T, Itoh T. TENOR: database for comprehensive mRNA-Seq experiments in rice. Plant Cell Physiol. 2015;

7. Lu TT, Zhu CR, Lu GJ, Guo YL, Zhou Y, Zhang ZY, Zhao Y, Li WJ, Lu Y, Tang WH, et al. Strand-specific RNA-seq reveals widespread occurrence of novel cis-natural antisense transcripts in rice. BMC Genomics. 2012;13:721.

8. Wang H, Chung PJ, Liu J, Jang IC, Kean MJ, Xu J, Chua NH. Genome-wide identification of long noncoding natural antisense transcripts and their responses to light in Arabidopsis. Genome Res. 2014;24(3):444-53.

9. Yu X, Yang J, Li XR, Liu XX, Sun CB, Wu FJ, He YK. Global analysis of cisnatural antisense transcripts and their heat-responsive nat-siRNAs in Brassica rapa. BMC Plant Biol. 2013;13:208

10. Swiezewski S, Liu F, Magusin A, Dean C. Cold-induced silencing by long antisense transcripts of an Arabidopsis Polycomb target. Nature. 2009;462(7274):799-802

11. Jabnoune M, Secco D, Lecampion C, Robaglia C, Shu QY, Poirier Y. A Rice cis-natural antisense RNA acts as a translational enhancer for its cognate mRNA and contributes to phosphate homeostasis and plant fitness. Plant Cell. 2013;25(10):4166-82.

12. Yoshida S, Forno AD, Cock HJ, Gomez AK. Laboratory manual for physiological studies of Rice. 3rd ed. Manila: The International Rice Research Institute (IRRI); 1976.

13. Martin M. Cutadapt removes adapter sequences from high-throughput sequencing reads. 2011;17:10-2.

14. Ohyanagi $H$, Tanaka $T$, Sakai H, Shigemoto $Y$, Yamaguchi $K$, Habara T, Fujii $Y$, Antonio BA, Nagamura Y, Imanishi T, et al. The Rice annotation project database (RAP-DB): hub for Oryza sativa Ssp. japonica genome information. Nucleic Acids Res. 2006;34(Database issue):D741-4.

15. Langmead B, Trapnell C, Pop M, Salzberg SL. Ultrafast and memory-efficient alignment of short DNA sequences to the human genome. Genome Biol. 2009;10(3):R25. 
16. Li H, Handsaker B, Wysoker A, Fennell T, Ruan J, Homer N, Marth G, Abecasis $G$, Durbin R. The sequence alignment/map format and SAMtools. Bioinformatics. 2009;25(16):2078-9.

17. Trapnell C, Pachter L, Salzberg SL. TopHat: discovering splice junctions with RNA-Seq. Bioinformatics. 2009;25(9):1105-11.

18. Mortazavi A, Williams BA, Mccue K, Schaeffer L, Wold B. Mapping and quantifying mammalian transcriptomes by RNA-Seq. Nat Methods. 2008;5(7):621-8.

19. Oono Y, Yazawa T, Kawahara Y, Kanamori H, Kobayashi F, Sasaki H, Mori S, Wu J, Handa H, Itoh T, et al. Genome-wide transcriptome analysis reveals that cadmium stress signaling controls the expression of genes in drought stress signal pathways in rice. PLoS One. 2014;9(5):e96946.

20. Yamada K, Lim J, Dale JM, Chen HM, Shinn P, Palm CJ, Southwick AM, Wu $\mathrm{HC}$, Kim C, Nguyen M, et al. Empirical analysis of transcriptional activity in the Arabidopsis genome. Science. 2003;302(5646):842-6.

21. Oono $Y$, Kawahara $Y$, Kanamori H, Mizuno H, Yamagata $H$, Yamamoto $M$, Hosokawa S, Ikawa H, Akahane I, Zhu Z, et al. mRNA-seq reveals a comprehensive transcriptome profile of rice under phosphate stress. Rice. 2011;4(2):50-65.

22. Wakasa Y, Oono Y, Yazawa T, Hayashi S, Ozawa K, Handa H, Matsumoto T, Takaiwa F. RNA sequencing-mediated transcriptome analysis of rice plants in endoplasmic reticulum stress conditions. BMC Plant Biol. 2014;14:101.

23. Shim D, Hwang JU, Lee J, Lee S, Choi Y, An G, Martinoia E, Lee Y. Orthologs of the class A4 heat shock transcription factor HsfA4a confer cadmium tolerance in wheat and rice. Plant Cell. 2009;21(12):4031-43.

24. Schofield RA, Bi YM, Kant S, Rothstein SJ. Over-expression of STP13, a hexose transporter, improves plant growth and nitrogen use in Arabidopsis thaliana seedlings. Plant Cell Environ. 2009;32(3):271-85.

25. Holmberg N, Bulow L. Improving stress tolerance in plants by gene transfer. Trends Plant Sci. 1998;3(2):61-6.

26. Torresschumann S, Godoy JA, Pintortoro JA. A probable lipid transfer protein gene is induced by Nacl in stems of tomato plants. Plant Mol Biol. 1992;18(4):749-57.

27. Frova $C$. The plant glutathione transferase gene family: genomic structure, functions, expression and evolution. Physiol Plant. 2003;119(4):469-79.

28. Zhao Y, Cheng S, Song Y, Huang Y, Zhou S, Liu X, Zhou DX. The interaction between rice ERF3 and WOX11 promotes crown root development by regulating gene expression involved in cytokinin signaling. Plant Cell. 2015;27(9):2469-83.

29. Li G, Liang W, Zhang X, Ren H, Hu J, Bennett MJ, Zhang D. Rice actinbinding protein RMD is a key link in the auxin-actin regulatory loop that controls cell growth. Proc Natl Acad Sci U S A. 2014;111(28):10377-82.

30. Lu C, Jeong DH, Kulkarni K, Pillay M, Nobuta K, German R, Thatcher SR, Maher C, Zhang L, Ware D, et al. Genome-wide analysis for discovery of rice microRNAs reveals natural antisense microRNAs (nat-miRNAs). Proc Natl Acad Sci U S A. 2008;105(12):4951-6.

\section{Submit your next manuscript to BioMed Central and we will help you at every step:}

- We accept pre-submission inquiries

- Our selector tool helps you to find the most relevant journal

- We provide round the clock customer support

- Convenient online submission

- Thorough peer review

- Inclusion in PubMed and all major indexing services

- Maximum visibility for your research

Submit your manuscript at www.biomedcentral.com/submit
Biomed Central 